\title{
Diffuse alopecia of the scalp in borderline- lepromatous leprosy in an Indian patient
}

\author{
SELVASEKAR ABRAHAM, GIGI J. EBENEZER \& \\ K. JESUDASAN \\ Schieffelin Leprosy Research \& Training Centre, Karigiri, NAA Dt., \\ Tamil Nadu, South India-632 106
}

Accepted for publication 14 August 1997

Summary A case of borderline-lepromatous leprosy exhibiting alopecia of the scalp along with lepromatous lymphadenitis of suboccipital lymphnode is reported. To our knowledge generalized leprous alopecia of the scalp with lepromatous lymphadenitis of the suboccipital node is a rare occurrence in female Indian patients.

\section{Introduction}

Alopecia is a common manifestation of leprosy especially in a few specific areas such as eyebrows, eyelashes and skin lesions. The scalp is usually spared. Cochrane in $1964^{1}$ stated that in certain races, particularly the Mongolian, leprous alopecia of the scalp is fairly common. It is rare in Africans, and has never been reported in Indians.

In this paper an Indian borderline-lepromatous leprosy patient with generalized alopecia of the scalp is reported.

\section{Case report}

A 64-year-old female reported to one of the village clinics of the Schieffelin Leprosy Research and Training Centre (SLR \& TC), Karigiri in November 1974, and on examination was diagnosed as having borderline-lepromatous leprosy. Dapsone, 5-mg daily, was prescribed and she was advised to take the drug regularly. But she never turned up for treatment and was next seen in the outpatient department of SLR \& TC, Karigiri in May 1990 after a period of 16 years with an acute infection of the right ring finger. She was treated with antibiotics and the abscess was incised and drained. She had never had regular antileprosy therapy, and therefore her disease had progressed. She was again prescribed dapsone, 50-mg daily for one month and advised to attend the leprosy clinic near her home and to receive antileprosy treatment regularly. She was never seen again until November 1995 when she reported to the skin clinic, Gudiyatham, with complaints of stuffiness and a blood-stained discharge from the nose of one-year's duration. 
On examination there was diffuse infiltration of the skin over almost the entire trunk and extremities and the skin was dry and scaly. Scratch marks with multiple abrasions and postinflammatory depigmented scars were found over the upper extremities. Bilateral madarosis and marked generalized alopecia of the scalp was present (Figure 1(a)). There was also loss of sensation in all four extremities and a trophic ulcer in the left foot, along with left foot drop and lagophthalmos on the right side. The suboccipital lymphnode on the left side was enlarged and measured $1.2 \mathrm{~cm} \times 0.8 \mathrm{~cm}$. It was discrete, freely mobile and rubbery in consistency. A clinical diagnosis of lepromatous leprosy with generalized alopecia of the scalp and suboccipital lymphadenitis was made.
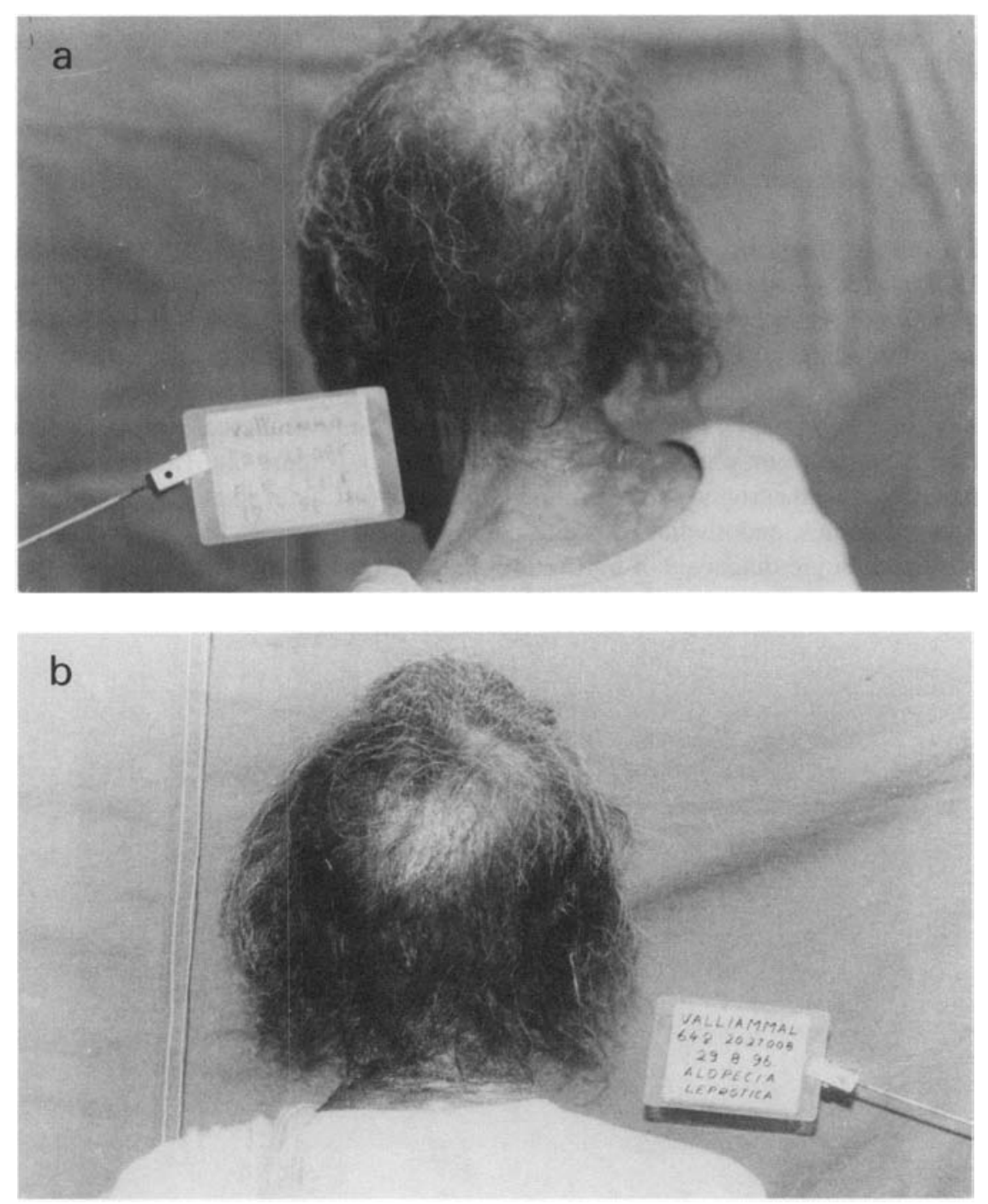

Figure 1. (a) Clinical photograph showing significant loss of hair throughout the scalp. (b) Clinical photograph of the same patient showing appreciable growth of scalp hair after 6 pulses of MDT-MBR. 
Routine laboratory investigations, including a chest X-ray were normal. Special investigations like renal and liver function tests were within normal limits. Hormonal assays which included serum testosterone, serum thyroxin $\left(\mathrm{T}^{4}\right)$, thyroid stimulating hormone (TSH) and free thyroxin concentration did not show any abnormal levels. HIV and VDRL were negative. Abdominal ultrasonogram was normal.

Dermatological conditions like scabies, pediculosis, tinea, pyogenic infections, telogen effluvium, lichen planus, lupus erythematosus, sarcoidosis and scleroderma were ruled out. The patient was also not on any drugs that have been known to cause alopecia.

Skin smears showed a bacterial index (BI) of 3.80+. A biopsy of the lesion on the right buttock was done. Since she was found to have alopecia, biopsy of the scalp was also done. The lymph node from the suboccipital region was excised. She was started on multidrug therapy-multibacillary regimen (MDT-MBR). Six months later there was an appreciable growth of scalp hair (Figure 1(b)).

\section{Histopathological examination}

The skin biopsy, the scalp biopsy and the lymph node were fixed in $10 \%$ buffered neutral formalin for over $48 \mathrm{hr}$; processed for paraffin sections; 5- $\mu \mathrm{m}$ sections were cut and stained both with hematoxylin eosin stain and modified Fite's stain for acid-fast bacilli (AFB).

Histopathological examination of the skin showed epidermis with minimal epithelial hyperplasia and obliteration of rete ridges. There were discrete granulomas in the dermis composed of macrophages mixed with numerous diffusely scattered lymphocytes, dilated lymphatic vessels were present throughout the lesion (Figure 2). Dermal nerves were surrounded by the macrophage granuloma. The acid-fast stain showed clumps of bacilli inside nerve bundles, endothelial cells and macrophages.

A histopathologic diagnosis of borderline-lepromatous leprosy was made.

The scalp lesion showed epidermis with some epithelial hyperplasia. There was marked

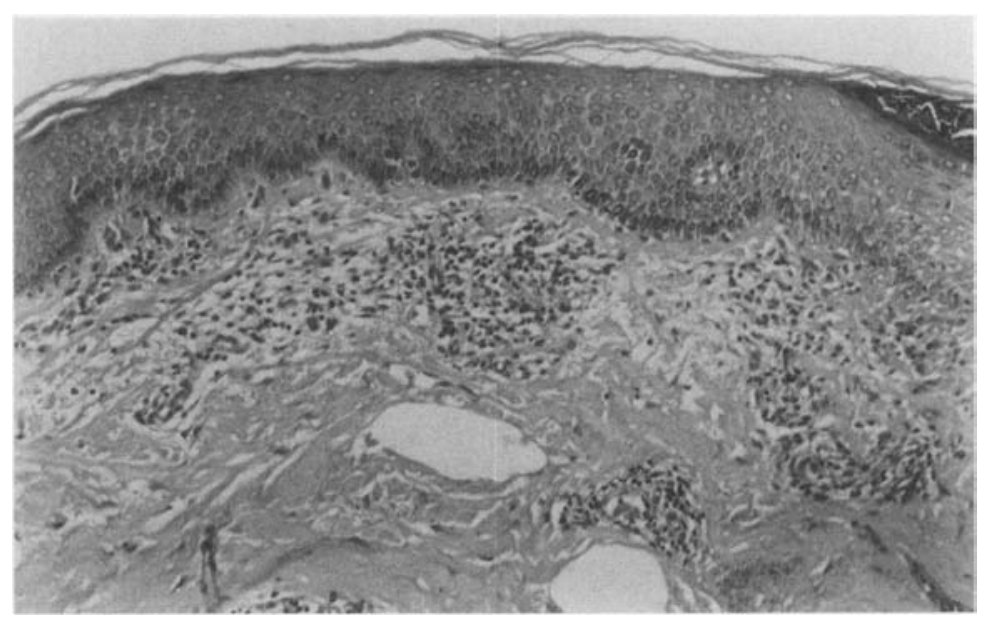

Figure 2. Photomicrograph of the skin lesion to show minimal epithelial hyperplasia and subepithelial granuloma composed of macrophages and lymphocytes. Two dilated lymphatics are also seen $(\mathrm{H} \& \mathrm{E} \times 300)$. 


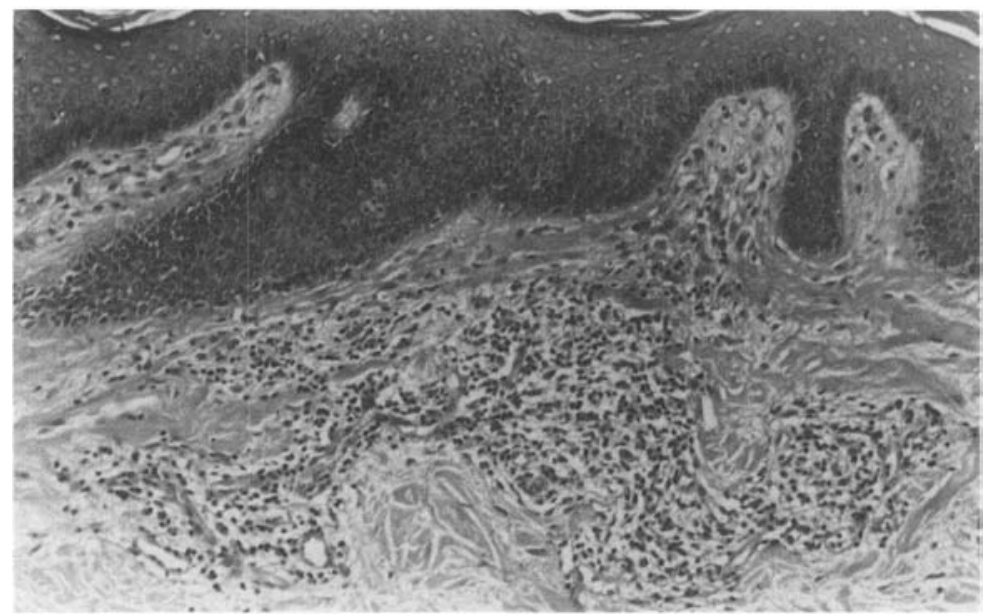

Figure 3. Photomicrograph of the scalp lesion to show hyperplasia of the epithelium and marked subepithelial infiltration with an admixture of lymphocytes and macrophages. An abortive hair follicle is also present $(\mathrm{H} \& \mathrm{E} \times 300)$.

reduction in the number of hair follicles. Discrete granulomas composed of macrophages and lymphocytes unevenly mixed together were seen in the subepithelial tissue (Figure 3). Mononuclear cell infiltration was seen around several atrophic hair follicles. Acid-fast stain showed clumps of bacilli inside macrophages and nerve bundles. Borderline-lepromatous leprosy of scalp was diagnosed.

Histopathological examination of lymph node showed normal architecture. Both the cortical and paracortical regions were infiltrated by small focal collections of foamy macrophages (Figure 4). Some of the macrophages had melanin pigment. Acid-fast stain showed bacilli in clumps inside the macrophages. The histopathologic appearance was consistent with lepromatous lymphadenitis.

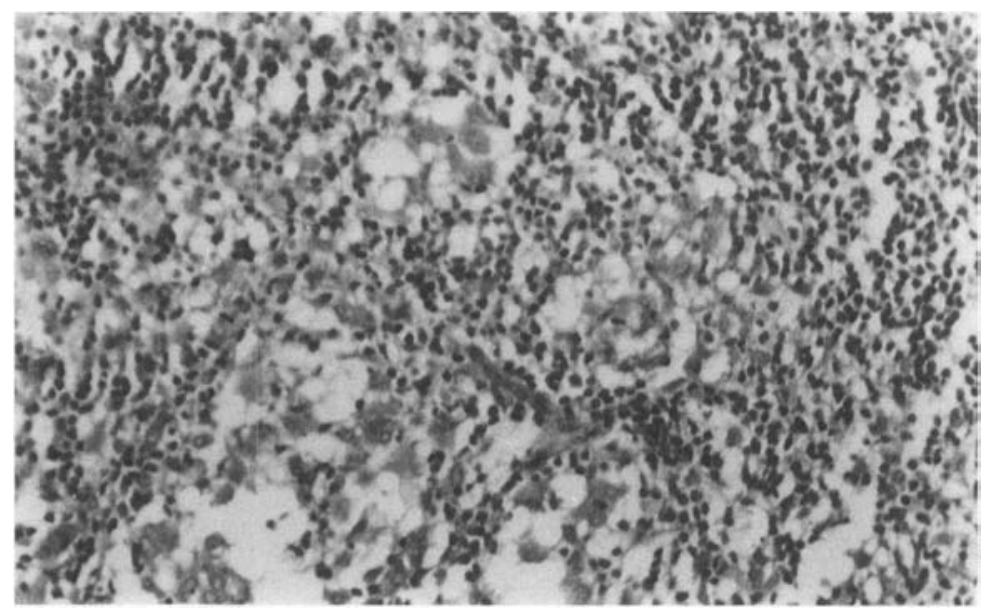

Figure 4. Photomicrograph to show lepromatous granuloma composed of foamy macrophages infiltrating lymph node tissue $(\mathrm{H} \& \mathrm{E} \times 600)$. 


\section{Discussion}

This patient, when she was first seen had a localized borderline-lepromatous disease which clinically degenerated into generalized subpolar lepromatous leprosy over a period of 21 years because she, inspite of all reminders, neglected to have adequate antileprosy therapy. She had generalized dry and scaly lesions almost all over the body and complete loss of hair over the eyebrows, and eyelashes. Histopathologic examination of the lesion over the buttock showed features diagnostic of borderline lepromatous disease.

In earlier reports patchy loss of hair had been recorded in tuberculoid, ${ }^{2}$ borderline ${ }^{3}$ and lepromatous leprosy patients. ${ }^{4,5}$ Generalized loss of hair in the scalp (Figure 1(a)) of this Indian female patient is a rare finding. Histopathologic examination of the scalp clearly showed infiltration of skin over the scalp by lepromatous granuloma and destruction of hair follicles, thus confirming that alopecia is produced by lepromatous disease. $M$. leprae grows in areas of the body where temperature is less than normal body temperature. Therefore lesions of lepromatous leprosy are abundantly seen in the cooler parts of the body such as the earlobes, eyebrows and nasal mucosa. Scalp has a higher temperature ${ }^{6,7}$ and therefore lepromatous leprosy lesions of the scalp are uncommon.

The suboccipital lymph node which drains the scalp, showed collections of foamy macrophages containing M. leprae (Fig. 4). Lepromatous lymphadenitis of the suboccipital lymph node is also a very rare finding as rare as the lepromatous involvement of the scalp.

\section{Acknowledgment}

The authors are thankful to Professor Charles K. Job, Consultant Pathologist, St Thomas Hospital and Leprosy Centre, Chettupattu for his encouragement and guidance. We also acknowledge Mr R. Soundararajan, Laboratory Technician, Mr M. Philip Benis, Social Worker, Mrs Rachel Charles, Librarian, and Ms Helen Jothi, Secretary for their assistance in the study.

\section{References}

${ }^{1}$ Cochrane RG. In Leprosy in theory and practice, edited by Cochrane RG and Davey TF 2nd Edition. Bristol John Wright and Sons Ltd. 1964: 266.

2 Edward VK, Edward S \& Shegaonkar S. Dry skin lesions with marked hair loss in a case of BL leprosy-a case report. Lepr Rev, 1996; 67: 141-144.

${ }^{3}$ Parikh AC, D'Souza NG, Chaulawala R \& Ganapati R. Leprosy lesions in the scalp. Lepr India, 1974; 46: 39-42.

4 Malaviya GN, Girdhar BK, Hussain S, Ramu G, Lavania RK \& Desikan KV. Scalplesion in a lepromatous leprosy patient—case report. Ind J Lepr, 1987; 59: 103-105.

5 Jayakumar J, Aschhoff M, Renuka G and Meyers WM. Involvement of scalp, axillae and groins in lepromatous leprosy. Ind J Lepr, 1992; 64: 541-544.

${ }^{6}$ Brand PW. Temperature variation and leprosy deformity. Int J Lepr, 1959; 27: 1-7.

${ }^{7}$ Sabin TD \& Ebner JD. Patterns of sensory loss in lepromatous leprosy. Int J Lepr, 1969; 37: 239-248. 